\title{
Identification of Common Genetic Markers of Paroxysmal neurological disorders using a network analysis approach
}

\begin{abstract}
Emerging data have established links between paroxysmal neurological disorders or psychiatric disorder, such as migraine, ataxia, movement disorders and epilepsy. Common gene signaturessuch as expression, protein interaction and the associated signalling pathways link genes in these associated disorders, with the object to predict unknown disease or risk genes. In this study, we used gene interaction networks to investigate common gene signatures associated with the above phenotypes.In total 19 candidate genes were used for making an interaction network which further revealed 39 associated genes (including KCNA1, SCN2A, CACNA1A, KCNM4, KCNO3, SCN1B and CACNB4) implicated in paroxysmal neurological disorders development and progression. The meta-regression analysis showed the strongest association of $S C N 2 A$ with genes involved in schizophrenia and neurodevelopmental disorders. Importantly, our analysis showed KCNMA1 as a common gene signature with a link to epilepsy, movement disorders and wide paroxysmal neurological presentations - with the greatest potential risk of being a disease gene in a paroxysmal or psychiatric disorder. Further gene interactions analysis is required to identify unidentified gene interactions which may be targets for future drugs development.
\end{abstract}

Keywords:Paroxysmal Neurological Disorder, Migraine, Ataxia, kinesigenic. 


\section{Introduction:}

Paroxysmal neurological disorder is a rapid recurrence or severity of symptoms, like seizure and tremor attacks. These symptoms can be observed in many clinical symptoms such as epilepsy, movement disorders, migraine and ataxia. Epilepsy is a common neurological condition that reflects neuronal hyper excitability arising from cellular and molecular mechanisms which are not yet completely understood [1]. Epilepsy does not only affect the individual patient, but it also has effects on the family and indirectly on the community [2]. According to results from the World Health Organization (WHO), there is at least 50 million people worldwide suffering from epilepsy [3]. The proportion of people with epilepsy living in developing countries is considered far greater and often no treatment is readily available for this condition [4].Epileptic patients could spend normal lives if proper treatment was provided. It is the most common severe neurological disorder with serious primary disease of the brain, which accounts for $1 \%$ of the global burden of neurological disease [5]. There is still a lack of authentic biomarkers for the detection of epilepsy, as well as for assessing the development and progression of epileptic condition [6]. With an estimated 2-3 million people living in the United States [7],six million in Europe [8] and at least 40 million in the developing world [9]. Epidemiological studies have previously showed that epileptic patients are 2.4 times more likely to develop migraine than their relatives without epilepsy [10]. The frequent co-existence of these paroxysmal neurological disorders is due to the abnormal underlying neuronal hyper-excitability[11]. In addition, both sporadic and familial epilepsy with infancy or early childhood on set are often associated with mutations in genes involved in pleiotropic paroxysmal neurological disorders, which include migraine/hemiplegic migraine, and paroxysmal movement disorders including kinesigenic/non-kinesigenic dyskinesia and episodicataxia.Co-existing episodic neurologic disorders frequently share molecular mechanisms which implicate abnormal function of ion channels and synaptic regulators involved in neuronal excitability and synaptic transmission [12].Familial hemiplegic migraine is an autosomal dominant (AD) disorder characterized bymigraine attacks marked by the occurrence of a transient hemiplegia during the aura. The three familial types of hemiplegic migraine 1, 2 and 3 (FHM1, FHM2, FHM3) are associated with heterozygous mutations in CACNA1A (MIM\# 601011),ATP1A2 (MIM \#182340) and SCN1A (MIM \#182389), respectively. A landmark genetic meta-analysis study pinpointed the association of several genetic poly- 
morphisms. In total 19 candidate genes were used in designingan interaction network to see if there is any common gene associated with multiple neurological presentations. We hypothesized that acommon gene signature and its associated signalling pathways would not only reveal its shared molecular mechanism, but also would serve as a potential early biomarker for diagnosis of epilepsy and other related paroxysmal neurological disorders.

\section{Material and Methods:}

Molecular pathways implicated in episodic ataxia and familial hemiplegic migraine intersects with pathways underlying other episodic neurological disorders. Mutations in the synaptic regulator genes PRRT2 and PNKD alter neurotransmitter release and are associated with infantile seizures, paroxysmal kinesigenic dyskinesia (PKD), FHM, or EA, and mutations in the brain glucose transporter SLC2Alcause paroxysmal exercise induced dyskinesia (PED), PKD, paroxysmal non-kinesigenic dyskinesia (PNKD) and EA [13]. Mutations in SCN8A have been reported in epilepsy, ataxia and paroxysmal dyskinesia (OMIM ID 118800) [14]. Mutations in $A T P 1 A 3$ can cause a wide array of neurological disorders and have also been described in association with EA [15 16]. ATP1A2 and SCN1A are crucial in regulating glutamatergic synaptic transmission and presynaptic action potentials, respectively, and mutations in these genes are implicated in FHM2 and FHM3 and epilepsy [17 18].

2.1. Retrieval of common genes associated with disease:

Relevant research articles were studied and information about genes associated with epilepsy, and paroxysmal presentations of ataxia, migraine, and movement disorders was collected. Disease names were used as key words to search on PubMed (https://www.ncbi.nlm.nih.gov/pubmed/27300529) and other literature databases. Severalstudies were included in the analysis using a bioinformatics approach to choose the common gene signatures for the above diseases. DisGeNET[19] was used asa discovery platform is integrating information on gene-disease associations (GDAs) from several public data sources and literature pertaining to gene expression, biomarker, variant-diseaseassociation, singlenucleotide 
polymorphism and clinical phenotype association with corresponding diseases. The latest version of DisGeNET (v4.0) contains 429036 associations between 15093 diseases and 17381 genes[20]. A Venn diagram was plotted using online venny 2.1.0 tool (http://bioinfogp.cnb.csic.es/tools/venny/) to visualize common gene signatures.

\subsection{Functional enrichment analysis:}

Gene enrichment analysis was carried out onWebGestalt 3.0 stand-alone software tool [21]. The prediction of clinical phenotype enrichment and biological pathways is carried out with the help ofWebGestalttool. The new user-friendly output interface and the GOview tool allow interactive and efficient exploration and comparison of enrichment results. Thus, WebGestalt 2017 enables more comprehensive, powerful,flexible and interactive functional enrichment analysis. It is freely available at http://www.webgestalt.org.

\subsection{Construction of Network and analysis:}

All the retrieved genes were used as input for the GeneManiawhich is a flexible, user-friendly cytoscape plugin for generating hypothesis about gene function, analysing gene list and prioritizing genes for functional assays[22]. GeneMania takes gene symbols or NCBI gene IDs as input to draw network. All 19 genes symbols werevalidatedby the GeneMania and were visualized as network. The network consists of 39 genes. The black nodes are our query genes and remaining grey nodes are relevant genes having most connectivity with our query genes. In the Gene-Disease Network, diseases were connected with the associated candidate targets. The corresponding diseases of potential genes were collected by DisGeNET, and the obtained interactions between diseases and genes were applied further for building the Gene-Disease Network. Different type of interactions like physical interactions, co-expression, shared protein domains, pathways etc. are represented by different colourededges. In this bilateral network, the 'nodes' represented the protein targets, diseases, and 'edges' represented the interactions of Gene-Gene or Gene-Disease. The networks were constructed using Cytoscape v3.6.1 (https://cytoscape.org/). 


\section{Results:}

Candidate genes, responsible for epilepsy, ataxia, migraine, and movement disorders, were collected through a literature review and by using DisGeNET. Most of the listed genes-disease associations have already been validated in our laboratory at the Institute of Neurology, University College London as part of a gene panel for paroxysmal neurological disorders (Figure 1). These genes were also confirmed with GeneMAINA which provides the total number of reported genes that have genetic association with the above diseases. After the first stage of text analytics using literature databases, the number of genes identified was 39 . 

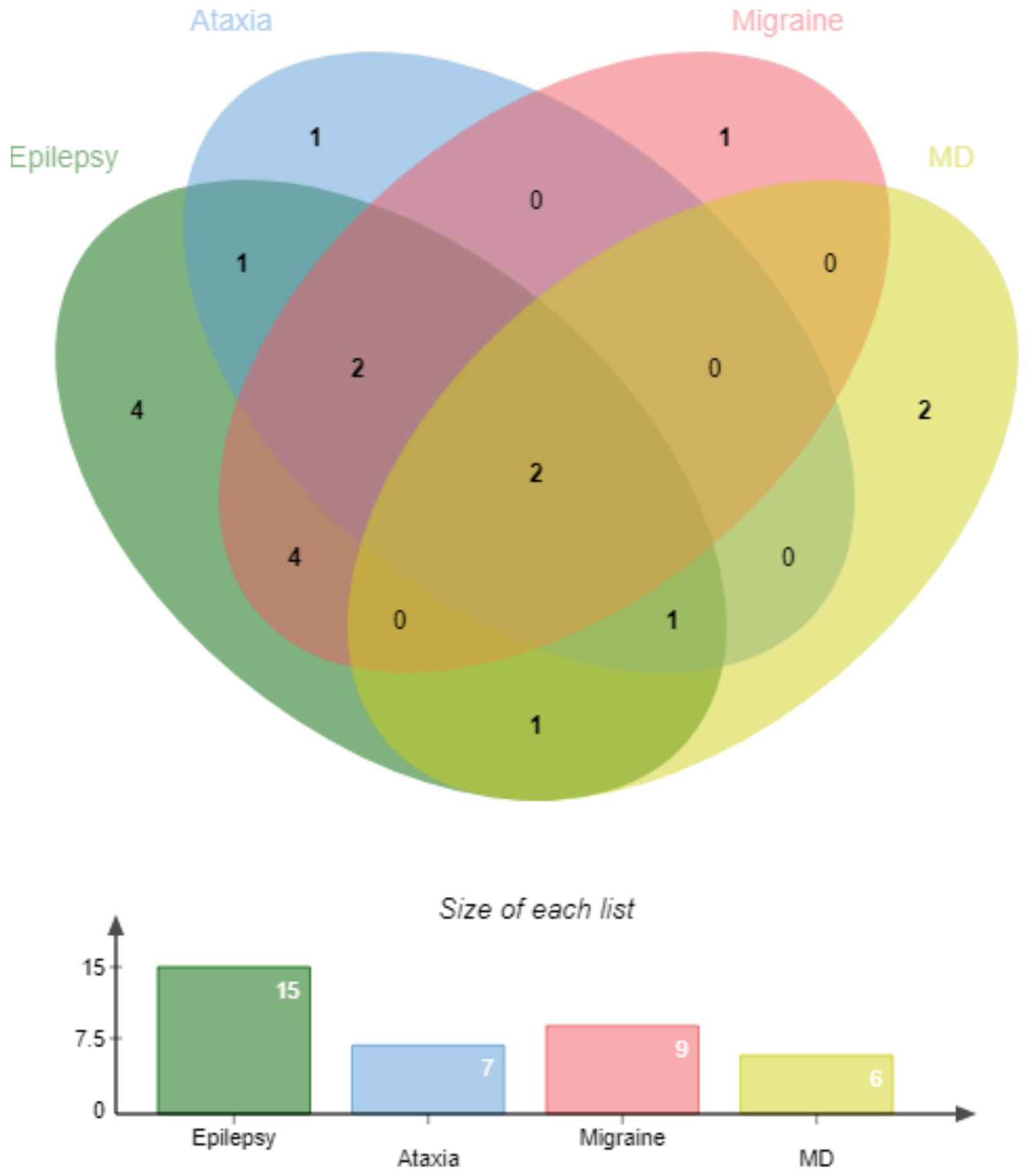

Number of elements: specific (1) or shared by 2, 3,.. lists

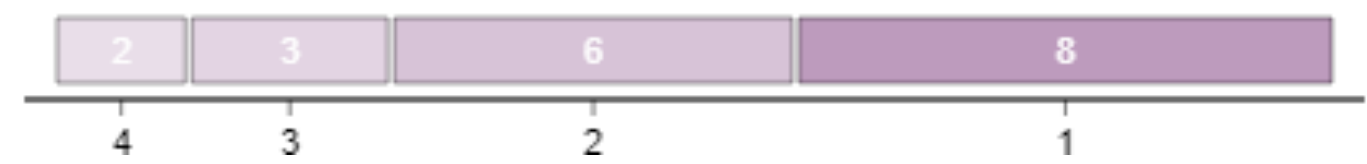

Fig.1. Venn diagram showing different diseases sharing associated genes. 
In order to find the common genes associated with epilepsy, ataxia, migraine and movement disorders, we collected a list of disease using DisGeNET database v4.0 and correlated their affinity by plotting a Venn diagram (Figure 1). There were a total of 39 genes in which 14 of the total genes associated with epilepsy. These results were in consensus with previous studies reporting common risk factors involved in above mentioned diseases. Furthermore, it gave the incentive for the characterization of these common gene signatures.

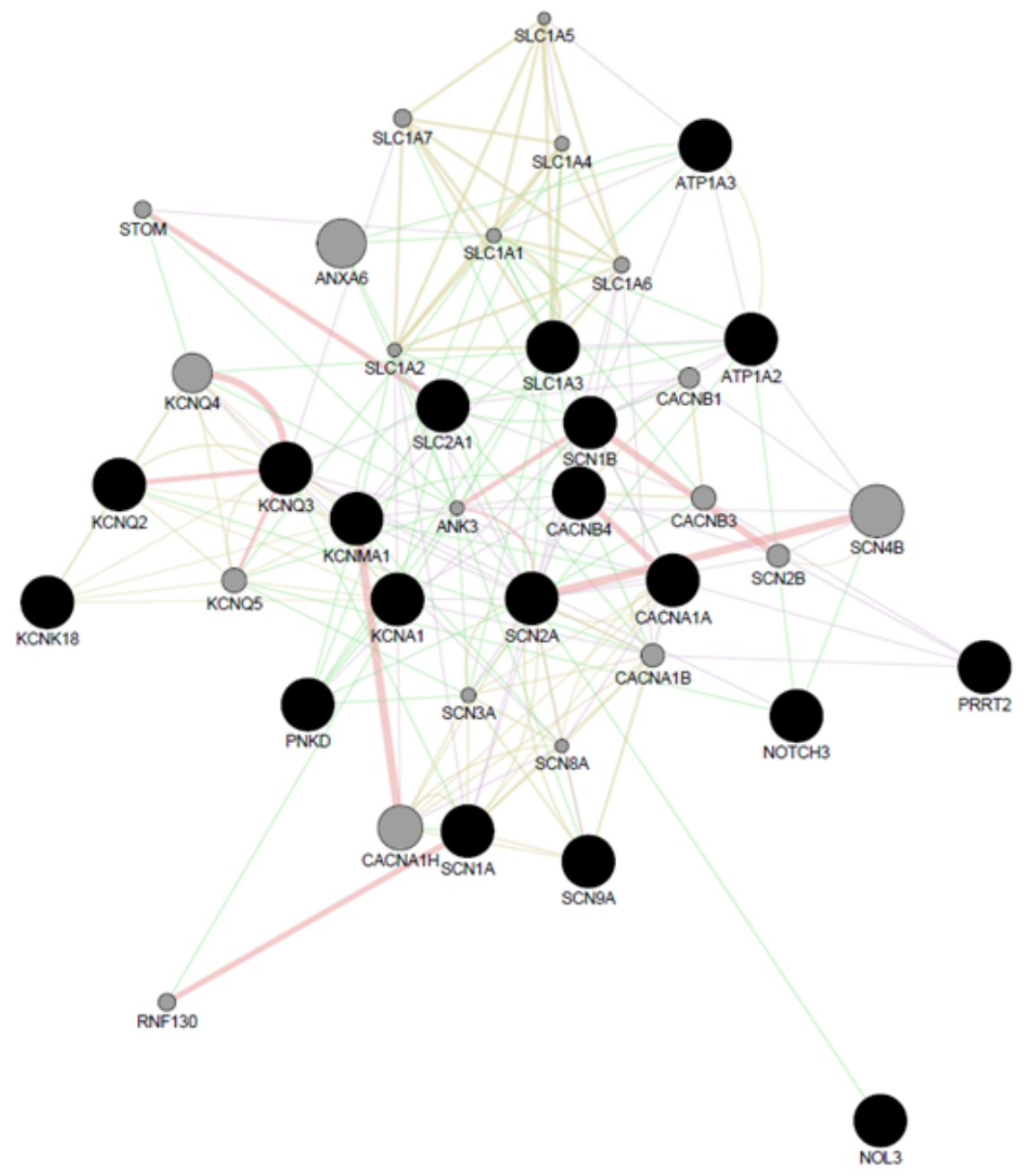

Fig.2. General Protein-Protein Network showing different interactions of candidate genes

\section{Discussion:}


Genetic associations with specific disorders are meaningful only if they contribute towards disease development and progression. The investigation of a gene signature that was common to epilepsy, ataxia, migraine and movement disorders across clinical phenotype, physiology, biological processes and pathways was performed. We reflect that this top-down approach would help in the better understanding of the biological significance of a common gene signature. The predicted results of the correlation of physiology level with clinical phenotypes showed (Figure 3) an enrichment of genes associated with biological processes (Red), cellular components (Blue) and molecular functions (Green), according to the analysis by WebGestalt.
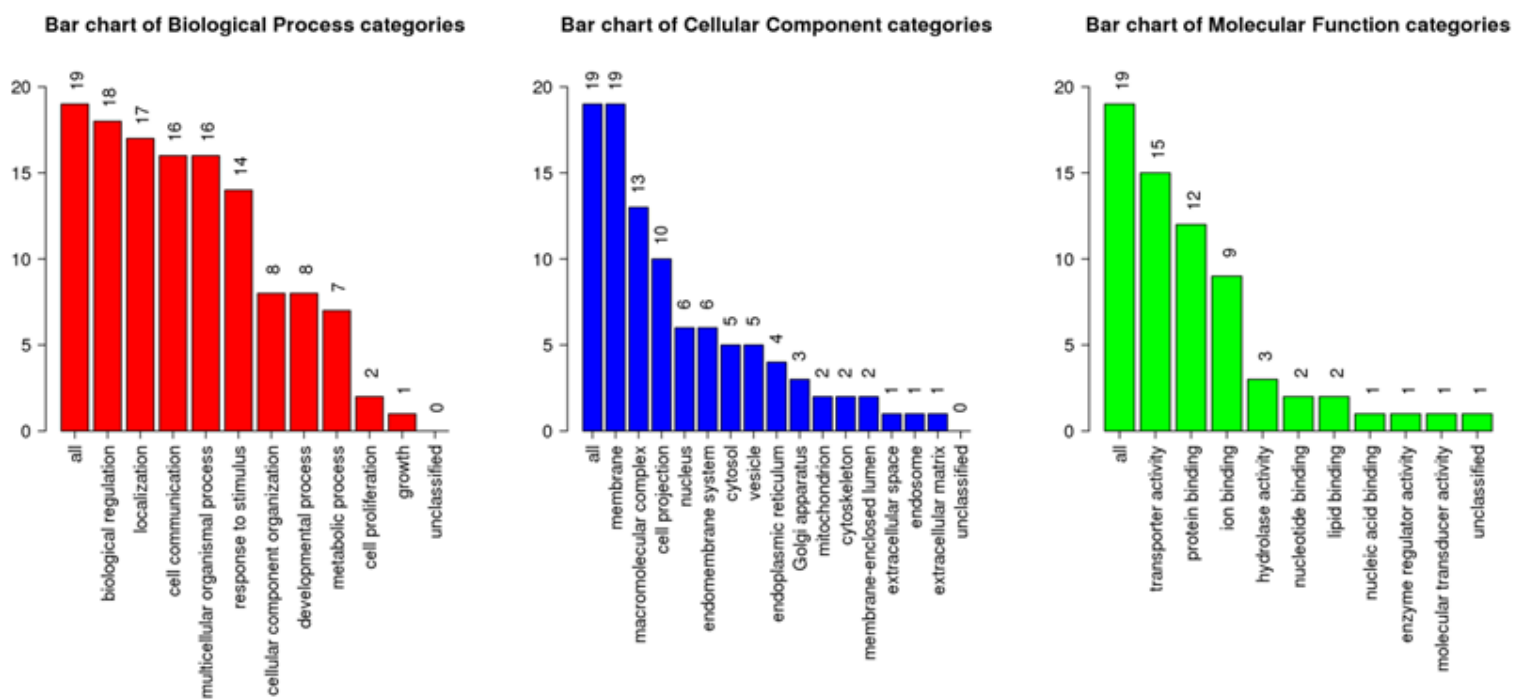

Fig.3.(Red) Biological process (Blue) Cellular component (Green) Molecular function.

Afterwards, gene prioritization analysis was performed with the help of Top Genetool, which aids in the ranking of the candidate genes by profiling across multiple genomic data sources and integrate this heterogeneous information into global ranking information. All 39 genes were ranked and their protein-protein interaction network was created using Cytoscape and based on gene ontology, literature review, experimental observations, and gene expression neighbourhoods (Figure 4). The subsequent analysis revealed that KCNA1, SCN2A, CACNA1A, KCNM4, KCNO3, SCN1B and CACNB4 are important nodes in the network with many interacting partners. One of the polymorphism in candidate genes, KCNMA1, has previously been reported for its association with neurodegenerative disorders, including Alzheimer's disease, as shown by the meta-analysis results from the AlzGene database (Figure 5). In conclusion, our re- 
sults identified ranked candidate genes that were implicated in epilepsy, ataxia, migraine and movement disorders but also involved in other neurological disorders.

Mutations in KCNMA1 causes functional change in ion channels, excitability of neurons with the release of impaired neurotransmitters that should be classified on the basis of pathophysiology as channelopathies (calcium-activated potassium channel subunit alpha-1 and voltage-gated sodium channel type 8) [23]. Most common types of epilepsy and their different loci has been identified through genome-wide association study and meta-analysis approach. Study findings suggested that some loci might have specific association with epilepsy subtype.SCN1A has clear association with epilepsy progression [24]. Frequency of SCN2A mutations responsible for Epilepsy and neurological disorders is reported to be highest among Danish population.SCN2A (sodium voltage gated channel) variation is highly associated with epilepsy spectrum and majority of neurodegenerative disorders [25]. Widespread pathogenic variants in SCN2A and KCNA1 (Potassium gated channel) are responsible for episodic ataxia whose episodes lasts for seconds to minutes [26]. In response to pathophysiological approaches blockers such as ion channels is a diagnostic and potential drug target clue for potassium and sodium ion channel mutations. Collective and intense studies are required to describe shared therapeutic protocols [27]. 


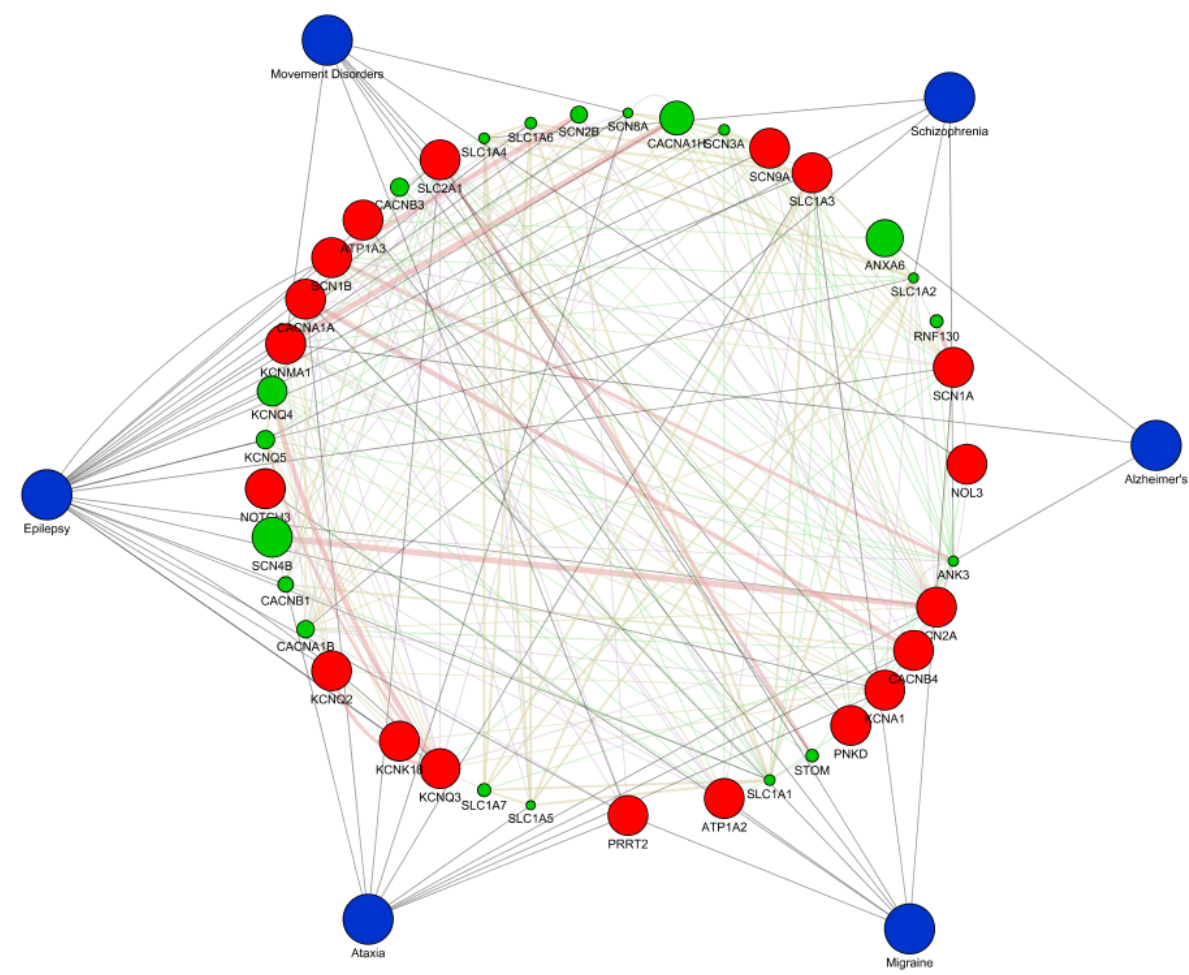

Fig.4. The network shows Interaction between genes associated with 6 diseases (epilepsy, ataxia, migraine, Alzheimer, schizophrenia and movement disorders). The genes are shown as red and green nodes and the diseases as blue.

AlzGene meta-analysis for KCNMA1 (rs35807): G vs. C

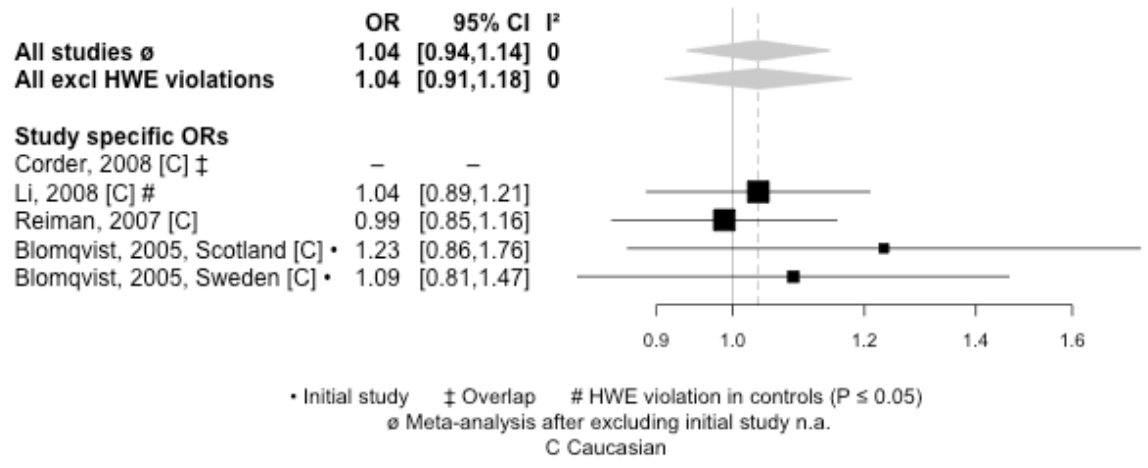

Fig. 5. Forest plot showing KCNMA1 gene polymorphism association with Alzheimer disease.

\section{Conclusion:}


Neurological disorders are major causes of physical and intellectual disability and causing many severe and chronic diseases that are hard to diagnose and differentiate. Epilepsy, migraine, ataxia and movement disorders are some common neurological disorders that remain for lifetime. Our network analysis showed common genes associated with the above mentioned neurological disorders that will help in early diagnosis and could provide information for genetic counselling. It could be proved as a useful tool for drug designing of mentioned neurological conditions.

\section{References:}

1. Lossin, C., Wang, D. W., Rhodes, T. H., Vanoye, C. G., \& George Jr, A. L. (2002). Molecular basis of an inherited epilepsy. Neuron, 34(6), 877-884.

2. England, M. J., Liverman, C. T., Schultz, A. M., \& Strawbridge, L. M. (2012). Epilepsy across the spectrum: Promoting health and understanding.: A summary of the Institute of Medicine report. Epilepsy \& Behavior, 25(2), 266-276.

3. World Health Organization. WHO press; (2006). Neurological disorders: public health challenges. Available: http://www.who.int/mental health/neurology/neurodiso/en/index.html

4. Reynolds, E. H. (2001). ILAE/IBE/WHO Global Campaign "Out of the Shadows": global and regional developments. Epilepsia, 42(8), 1094-1100.

5. Lhatoo, S. D., \& Sander, J. W. (2005). Cause-specific mortality in epilepsy. Epilepsia, 46, 36-39.

6. Engel, J. (2011). Biomarkers in epilepsy: introduction. Biomarkers in medicine, 5(5), 537-544.

7. Hirtz, D., Thurman, D. J., Gwinn-Hardy, K., Mohamed, M., Chaudhuri, A. R., \& Zalutsky, R. (2007). How common are the "common" neurologic disorders?. Neurology, 68(5), 326-337.

8. Meyer, A. C., Dua, T., Ma, J., Saxena, S., \& Birbeck, G. (2010). Global disparities in the epilepsy treatment gap: a systematic review. Bulletin of the World Health Organization, 88, 260-266.

9. Theodore, W. H., Spencer, S. S., Wiebe, S., Langfitt, J. T., Ali, A., Shafer, P. O., ... \& Vickrey, B. G. (2006). Epilepsy in North America: a report prepared under the auspices of the global campaign against epilepsy, the International Bureau for Epilepsy, the International League Against Epilepsy, and the World Health Organization. Epilepsia, 47(10), 1700-1722.

10. Joutel, A., Bousser, M. G., Biousse, V., Labauge, P., Chabriat, H., Nibbio, A., ... \& Lathrop, G. M. (1993). A gene for familial hemiplegic migraine maps to chromosome 19. Nature genetics, 5(1), 40.

11. Lipton, R. B., Ottman, R., Ehrenberg, B. L., \& Hauser, W. A. (1994). Comorbidity of migraine: the connection between migraine and epilepsy. Neurology, 44(10 Suppl 7), S28-32.

12. Aurora, S. K., Cao, Y., Bowyer, S. M., \& Welch, K. M. A. (1999). The occipital cortex is hyperexcita- 
ble in migraine: experimental evidence. Headache: The Journal of Head and Face Pain, 39(7), 469-476.

13. Gardiner, A. R., Bhatia, K. P., Stamelou, M., Dale, R. C., Kurian, M. A., Schneider, S. A., ... \& Valente, E. M. (2012). PRRT2 gene mutations: from paroxysmal dyskinesia to episodic ataxia and hemiplegic migraine. Neurology, 79(21), 2115-2121.

14. Gardiner, A., Bhatia, K., Stamelou, M., Dale, R., Kurian, M., Schneider, S., ... \& Valente, E. (2013). PRRT2 gene mutations: From paroxysmal dyskinesia to episodic ataxia and hemiplegic migraine. Headache, 53(4), 693-694.

15. N'Gouemo, P. (2011). Targeting BK (big potassium) channels in epilepsy. Expert opinion on therapeutic targets, 15(11), 1283-1295.

16. Haut, S. R., Bigal, M. E., \& Lipton, R. B. (2006). Chronic disorders with episodic manifestations: focus on epilepsy and migraine. The Lancet Neurology, 5(2), 148-157.

17. De Boer, H. M., Mula, M., \& Sander, J. W. (2008). The global burden and stigma of epilepsy. Epilepsy \& behavior, 12(4), 540-546.

18. Du, W., Bautista, J. F., Yang, H., Diez-Sampedro, A., You, S. A., Wang, L., ... \& Richerson, G. B. (2005). Calcium-sensitive potassium channelopathy in human epilepsy and paroxysmal movement disorder. Nature genetics, 37(7), 733.

19. Piñero, J., Queralt-Rosinach, N., Bravo, A., Deu-Pons, J., Bauer-Mehren, A., Baron, M., ... \& Furlong, L. I. (2015). DisGeNET: a discovery platform for the dynamical exploration of human diseases and their genes. Database, 2015.

20. Ni, P., Wang, J., Zhong, P., Li, Y., Wu, F., \& Pan, Y. (2018). Constructing Disease Similarity Networks Based on Disease Module Theory. IEEE/ACM transactions on computational biology and bioinformatics.http://bioinfogp.cnb.csic.es/tools/venny/

21. Wang, J., Vasaikar, S., Shi, Z., Greer, M., \& Zhang, B. (2017). WebGestalt 2017: a more comprehensive, powerful, flexible and interactive gene set enrichment analysis toolkit. Nucleic acids research, 45(W1), W130-W137.

22. Warde-Farley, D., Donaldson, S. L., Comes, O., Zuberi, K., Badrawi, R., Chao, P., ... \& Maitland, A. (2010). The GeneMANIA prediction server: biological network integration for gene prioritization and predicting gene function. Nucleic acids research,38(suppl_2), W214-W220. https://genecards.weizmann.ac.il/v3/cgi-bin/carddisp.pl?gene=KCNMA1

23. Erro, R., Bhatia, K. P., Espay, A. J., \&Striano, P. (2017). The epileptic and nonepileptic spectrum of paroxysmal dyskinesias: channelopathies, synaptopathies, and transportopathies. Movement Disorders, 32(3), 310-318.

24. International League Against Epilepsy Consortium on Complex Epilepsies. Electronic address e-auea (2014). Genetic determinants of common epilepsies: a meta-analysis of genome-wide association studies. The Lancet Neurology, 13(9), 893.

25. Schwarz, N., Bast, T., Gaily, E., Golla, G., Gorman, K. M., Griffiths, L. R., ...\& Miranda, M. J. (2019). 
Clinical and genetic spectrum of SCN2A-associated episodic ataxia. European Journal of Paediatric Neurology, 23(3), 438-447.

26. Wolff, M., Johannesen, K. M., Hedrich, U. B., Masnada, S., Rubboli, G., Gardella, E., ...\&Afenjar, A. (2017). Genetic and phenotypic heterogeneity suggest therapeutic implications in SCN2A-related disorders. Brain, 140(5), 1316-1336.

27. Dilena, R., Striano, P., Gennaro, E., Bassi, L., Olivotto, S., Tadini, L., ...\&Fumagalli, M. (2017). Efficacy of sodium channel blockers in SCN2A early infantile epileptic encephalopathy. Brain and Development, 39(4), 345-348. 